\title{
A critical look at nursing observations
}

\author{
Janet Burroughs $^{1}$ and B.I. Hoffbrand ${ }^{2}$ \\ ${ }^{1}$ School of Nursing, Islington Health Authority and ${ }^{2}$ Department of Medicine, Whittington Hospital, \\ London, N19, UK.
}

Summary: Our experience has suggested that not only do nurses on hospital wards waste time making too many unnecessary clinical observations, but that it is very difficult to persuade them to modify the pattern of their practice.

We therefore conducted a survey by questionnaire among trained nurses in one health district of their everyday ward practice and attitudes to clinical observations. This showed that whilst a majority of nurses questioned believe in the importance of routine observations for patient care, there is a lack of consistency in the criteria and communication procedures used that must seriously limit the value of the information obtained by the individual nurse.

We believe that uncritical faith in routine observations is a barrier to rationalizing procedures to optimize efficient use of the professional skills of ward nurses in this regard. There is need for more thought to be given to the problem and in particular it is important that doctors play a more active role in decision making.

\section{Introduction}

The recording of clinical observations by nurses on patients admitted to hospitals is a traditional procedure for which there is often no logical basis. ${ }^{1,2}$ The making of accurate observations is a time-consuming and skilled activity whilst inappropriate use leads to poor performance on the part of the nurse and discomfort for the patient. Although we have found nurses apparently receptive to suggestions for rationalizing clinical observations, we have found ourselves unable to modify day-to-day practice on our own wards to any discernible degree. The current problems in nurse recruitment and loss from the profession, and the changes in deployment of nursing resources that will come with Project 2000 make the efficient use of nursing time essential. ${ }^{3}$ To these logistic reasons must be added the eternal need to maximize the professional skills of nurses and ensure the optimal care of patients. The major importance of achieving improvements in the deployment of nursing resources is well recognized in a recent report from the Department of Health. ${ }^{4}$ It is a pity, however, that time spent recording vital signs was not identified amongst the generally praiseworthy 'direct patient care' activities.

We have assessed the current practice and attitudes of nurses with regard to clinical observa-

Correspondence: J. Burroughs, B.A., S.R.N., R.M.N., O.N.C., Senior Nurse Research.

Accepted: 3 January 1990 tions. Our findings suggest that there are two major barriers to the introduction of effective guidelines to rationalize practice. One is the ingrained belief of many nurses in the value of routine observations. The other is the failure of clinicians to participate actively with nursing staff in deciding the observations needed in individual patients, what action needs taking in the light of agreed deviations, and when to reduce or stop making the observations.

\section{Methods}

A questionnaire was designed and sent to 24 wards within one inner London district health authority. A covering letter explained that we were interested in ward practice and the nurses opinion of it. Each ward that participated in the study received 6 questionnaires for completion by trained staff. Of the 144 sent out, 85 questionnaires were returned of which five were incorrectly filled in or from student nurses. The total sample analysed consisted therefore of 80 replies; $14(17.5 \%)$ came from sisters, $36(45 \%)$ from state registered nurses and $30(37.5 \%)$ from state enrolled nurses There were about equal replies from medical and surgical wards.

The questionnaire asked about current practice with regard to routine observations of temperature, pulse, respiration (TPR), blood pressure (BP) and finger-pricking blood glucose con- 
centration. 'Routine' was defined as those observations that are considered necessary because of the patients' diagnosis, treatment, or existing hospital or ward policy and applied to patients in that category irrespective of assessed need 'Policy' refers to actions that nurses must follow as a directive. (There are no hospital policies with regard to nursing observations and no written policies on any ward in the Whittington and Royal Northern Hospitals.)

\section{Results}

\section{Policy on routine observations}

Sixty six $(78 \%)$ nurses said a policy existed for the routine taking of observations in two situations. All new admissions were started on 4 hourly TPR and BP whilst half-hourly observations were to be made on all post-operative patients. Thirty eight $(48 \%)$ said the ward sister was responsible for making the policy as did about the same percentage of the sisters. Eight nurses believed that the policy was in writing.

\section{Deviations in observations recorded}

Sixty eight $(79 \%)$ nurses said that the nurse in charge decided what deviations should be reported, $7(8 \%)$ the allocated nurse and only $10(12 \%)$ said that this was decided by medical staff. About $25 \%$ indicated that all deviations from textbook norms were to be reported indicating a 'blanket policy' in operation. The means of indicating the decision about deviations to be reported was variably verbal or written in the care plan. No one method could be identified for a specific ward in regard to any of these procedures.

A majority $(65 \%)$ also said that deviations were reported to the nurse in charge, $5(6 \%)$ reported directly to the doctor whilst as many as $20(25 \%)$ did not respond to this question. A similar number also could not say who made the decision to change the frequency of observations. A majority $(64 \%)$ said that this was done by the allocated nurse or nurse in charge.

\section{Blood pressure recording}

Sixty seven $(84 \%)$ nurses said that BP was recorded routinely, daily in all hypertensive patients, or for 7 days post-operative. Four-hourly BP was recorded in hypotensive, hypertensive, post-operative or acutely ill patients and in those on hypertensive medication. Thirty-two $(\mathbf{4 0 \%})$ nurses used Phase IV and $48(60 \%)$ Phase V for diastolic BP. There was no agreement between nurses working in the same ward area.

\section{Blood glucose estimation}

Routine testing by BM Test Glycemie strips was used by half the nurses in insulin dependent diabetics, by $30(38 \%)$ in those on oral agents and by $17(21 \%)$ in diet controlled diabetics. There was no appreciable difference in practice between medical and surgical wards. Sixty four $(80 \%)$ said that a joint decision by medical and nursing staff was made about when testing was indicated, 58 $(73 \%)$ said that the testing was discontinued when blood sugars were acceptable and no fewer than 78 $(98 \%)$ said that abnormal readings led to alterations in treatment. Seventy seven also said that patients found the testing procedure unpleasant.

\section{Nurses' opinion about clinical observations}

Eighty three per cent of the nurses believed that a majority of patients on the ward need a daily TPR and $93 \%$ that recorded observations are used to make nursing decisions. Yet $36 \%$ felt that too much importance is attached to clinical observations and $54 \%$ that too much time is spent recording unnecessary observations. At the time the questionnaire was completed, $40 \%$ of the patients on the wards were having 4-hourly TPR observations, two thirds of whom were also having BP measurements. Over half the nurses said that frequent recording of observations provoked anxiety in the patients.

\section{Discussion}

This survey of trained nursing staff confirms previous studies ${ }^{1,2,5}$ and our personal experience that nursing observations are made largely as a routine procedure, unrelated to the perceived need of the individual patient. Activities that were once a habit, became a tradition and are now looked upon as 'policy'. We have observed this entire evolutionary process with the introduction of ward blood glucose testing. Since the completion of this study a directive has been issued to all wards, as the first hospital policy on observations, that this procedure should only be used repeatedly on a single patient at the request of a doctor. There has since been a $25 \%$ reduction in the supply of test strips from the District pharmacy, considerable money saved and many a sore finger prevented. We suspect diabetic control is none the worse.

It is apparent that a large majority of nurses believe routine observations to be needed on all patients and that these observations contribute to the patients' care. We believe there is an element of self-delusion here which explains the remarkable figure of almost $100 \%$ reporting that finding 
abnormal blood glucose levels leads to an alteration in treatment. This is certainly not our experience. One sister observed that nurses seem to like doing observations even when they have been stable for some time as it makes them feel secure. Another said that junior nurses and doctors keep patients on 4-hourly observations longer than is necessary for fear of missing something. A fear of the medico-legal implications of not recording vital signs frankly voiced to us over the years by often senior nurses seems a major obstacle to rationalizing the recording of observations.

Sixty-five per cent of the nurses said that observations are always recorded accurately, but the problems in maintaining quality control of ward BP and blood glucose measurement have been well documented. ${ }^{6,7}$ Our own nurses show a random distribution of Phase IV and Phase V diastolic BP measurers. We believe that the errors in recording observations increase sharply with the number that have to be made particularly in a setting where the rationale for them may not be clear. It is also apparent that there are deficiencies in communication with regard to deviations with $25 \%$ of the sample not apparently knowing to whom to report. We have noted a similar problem with routine

\section{References}

1. Mitchell, P.H. Concepts Basic to Nursing. McGraw-Hill, New York, 1977

2. Musallem, $H$. The nursing profession: ritualised, routinised or research-based? J Advanced Nursing 1979, 4: 90.

3. Department of Health Nursing Division. A Strategy for Nursing. A report of the steering committee. Crown Copyright, London, 1989.

4. Department of Health-NHS Management Consultancy Services/Nursing Division. Service Quality-a report on the activities of nursing staff on hospital wards. Crown Copyright, London, 1988. (Report No 1/88). urinalysis. $^{8}$

We believe it is important for individual ho: pitals to look critically at local practice in recordin observations. We would suggest that after nursin and medical assessments of newly admitte patients, only agreed observations are recorder the frequency, important deviations and action $t$ be taken being specified. These should relate to th individual problems and goals of the care plan s that this nursing activity has a rationale that shoul contribute to job satisfaction. Senior doctors mu be responsible for ensuring that their junior staff $d$ not misuse nursing time and expertise by asking $\mathrm{fc}$ inappropriate observations. Decisions to stop c reduce the frequency of observations shoul generally be the responsibility of the allocate nurse in line with the goal of securing indivi ualized care for patients and enhancing the profe sional role of the nurse through the encourageme of independent clinical judgement. ${ }^{3}$ It is importal to limit 'routine' observations to a few well define and validated situations. Above all we see a need 1 alter attitudes so that nurses and doctors ca recognize when professional time and skill is beir wasted on unnecessary and often patient-alarmir procedures.

5. Kilgour, D. \& Speedie, G. Taking the pressure off. Nursi Mirror 1985, 160: 39-40.

6. O'Brien, E., Fitzgerald, D. \& O'Malley, K. Blood pressu measurement: current practice and future trends. $\mathrm{Br} \mathrm{Med}$ 1985, 290: 729-734.

7. Hutchison, A. S. \& Shenkin, A. BM strips: how accurate a they in general wards? Diabetic Medicine 1984, 1: 225-22t

8. Hoffbrand, B.I. \& Watts, R. Missed proteinuria; the doct needs to be told. Br J Clin Pract (in press). 